This article is licensed under the Creative Commons Attribution-NonCommercial 4.0 International License (CC BY-NC) (http://www.karger.com/Services/OpenAccessLicense)

Usage and distribution for commercial purposes requires written permission.

\title{
Ocular Decompression Retinopathy with Macular Edema after Deep Sclerectomy with Intrascleral Implantation
}

\author{
Pablo Diaz Aljaro Jordi Loscos Arenas Laura Broc Iturralde \\ Marc Carbonell Puig Pau Romera Romero \\ Ophthalmology Department, Hospital Germans Trias i Pujol, Universitat Autònoma de \\ Barcelona, Badalona, Spain
}

\section{Keywords}

Glaucoma $\cdot$ Glaucoma filtrating surgery $\cdot$ Maculopathy · Deep sclerectomy

\begin{abstract}
We report a case of ocular decompression retinopathy (ODR) with macular edema, diagnosed by optical coherence tomography, after a deep sclerectomy (DS) with an intrascleral implant, resolved with medical therapy 6 months later. The medical literature reports that in $14 \%$ of patients suffering from ODR, a pars plana vitrectomy was required, and $15 \%$ of patients had a poor final visual acuity. An otherwise healthy 75 -year-old man with high myopia and a primary open-angle glaucoma, with previous intraocular pressure (IOP) of $24 \mathrm{~mm} \mathrm{Hg}$, underwent a DS with intrascleral implant without complications. The patient suffered postoperatively from ODR with macular edema that required medical therapy with nonsteroidal anti-inflammatory eye drops (Nepafenac ${ }^{\oplus} 0.3 \%$ ) for a period of 6 months. ODR is an infrequent complication that may occur after any surgical or medical procedure that causes a sudden IOP decrease. The presence of macular edema is only reported in $5 \%$ of cases and can occur in patients who report a decreased visual acuity, commonly associated with a retinal hemorrhage. We have described a case of ODR with macular edema after DS with intrascleral implant. Although ODR is considered to cause a low level of morbidity, in some patients this may not be the case.
\end{abstract}




\section{Introduction}

Ocular decompression retinopathy (ODR) is a rare complication that occurs after any ocular procedure that causes acute lowering of intraocular pressure (IOP). It is much more common after a trabeculectomy and the use of drainage devices than after deep sclerectomy (DS), as DS does not produce a sudden IOP decrease.

Different vascular and mechanical theories have been suggested. A very high preoperative IOP, hypotony, the use of antimetabolites, and a thin scleral thickness have all been considered as risk factors. This complication usually has no long-term consequences, but not in all cases. Previous literature reports that $14 \%$ of patients suffering from ODR required a pars plana vitrectomy and $15 \%$ of patients had a decreased final visual acuity [1].

\section{Case Report}

A 75-year-old highly myopic man suffering from primary open-angle glaucoma, with previous history of pars plana vitrectomy without complications due to an epiretinal membrane on his right eye, which reached a 0.4 visual acuity (Snellen chart at $5 \mathrm{~m}$ ) 10 months beforehand, underwent an uncomplicated DS with an intrascleral hydroxyethyl methacrylate implant (Esnoper ${ }^{\circledR}$ V-2000; AJL Ophthalmics, Álava, Spain). Mitomycin-C $0.02 \%(0.2 \mathrm{mg} / 0.1 \mathrm{~mL})$ was used for $1 \mathrm{~min}$. The axial length of this eye was $25.86 \mathrm{~mm}$ and pachymetry was $550 \mu \mathrm{m}$.

Both the intrascleral position of the implant and the exposure time to mitomycin-C were used, taking into consideration the anatomic and functional characteristics of the sclera of the myopic eye.

Preoperative IOP was $24 \mathrm{~mm} \mathrm{Hg}$ under maximum tolerable medical treatment. His postoperative IOP was $4 \mathrm{~mm} \mathrm{Hg}$ after $24 \mathrm{~h}, 12 \mathrm{~mm} \mathrm{Hg}$ in the first week, and $10 \mathrm{~mm} \mathrm{Hg}$ in the third week, but the visual acuity decreased to 0.15 (Snellen). Fundoscopy revealed multiple, small, round, intraretinal and preretinal hemorrhages on the posterior pole, which involved the optic disc, and macular edema which was confirmed by optical coherence tomography (OCT) (Zeiss Humphrey Systems, Dublin, CA, USA). No choroidal folds, retina striae, optic disc swelling, or retinal vessel tortuosity were observed (Fig. 1), ruling out a retinal venous occlusion.

Diagnosed with ODR and macular edema, the patient was treated with nonsteroidal antiinflammatory eye drops (Nepafenac ${ }^{\circledR} 0.3 \%$ ). Two months later the IOP was $16 \mathrm{~mm} \mathrm{Hg}$ without glaucoma treatment, but the OCT still revealed cystoid macular edema with no visual improvement (Fig. 2). It was not until 6 months later that the visual acuity reached its previous 0.4 (Snellen) and the cystoid macular edema resolved itself on the OCT (Fig. 3).

\section{Discussion}

ODR has been shown to occur primarily after a trabeculectomy or glaucoma drainage devices, but rarely after an uncomplicated DS [2]. It is more common in uveitic [3] and exfoliation glaucoma [4]. A multifocal hemorrhagic retinopathy, which is not accounted for by other processes, can also be seen. The multiple intraretinal, small, round hemorrhages would normally resolve themselves within a few days.

Mukkamala et al. [1] reviewed the current literature and found that ODR is not only described after glaucoma surgery; it has in fact also been observed after medical treatment for glaucoma, orbital decompression in Grave's orbitopathy, retinal surgery, phacoemulsification, 


\section{Case Reports in Ophthalmology}

or uveitis. Most of the patients are asymptomatic, except in cases where a macular hemorrhage or an edema is causing a decrease in visual acuity. This complication usually has no longterm consequences, but not in all cases. Although it was considered to pose a low morbidity rating in our patient, appropriate medical therapy was necessary for a period of 6 months.

There is no consensus regarding the pathophysiology, and several theories have been suggested. Mechanical theories suggest that an acute IOP decrease could result in anterior displacement of the vitreous, causing retinal hemorrhages. Scleral deformity could also play a role, reducing the axoplasmic flow through the lamina cribosa, hence resulting in an optic nerve edema. Vascular theory suggests that a sudden IOP decrease may reduce retinal arterial resistance, resulting in increased blood flow with impaired vascular autoregulation. Except for the fact that the patient was vitrectomized, both theories could be applied.

We have to consider that related literature on the subject reports preoperative IOP values of $45.7 \pm 16.9 \mathrm{~mm} \mathrm{Hg}$ as a major risk factor for ODR. However, in our case, the preoperative IOP was $24 \mathrm{~mm} \mathrm{Hg}$, granting more importance to the scleral rigidity and the vascular autoregulation dysfunction mechanism as the causing agents.

As OCT was not performed in most of the previous reports, a decrease in visual acuity has been attributed to macular blot hemorrhage. Mukkamala et al. [1] found macular edema in 5\% of the affected patients and Bui et al. [5] reported one case of ODR-associated macular edema diagnosed by OCT, arguing that macular edema could be underdiagnosed by the lack of imaging techniques in a higher percentage of eyes, explaining low visual acuity in the absence of hemorrhages. Differential diagnoses of retinal vascular disorders must be considered, but the sudden IOP decrease is almost always present in ODR. Valsalva retinopathy during extubation from general anesthesia and coagulopathies must also be considered. They were, however, excluded in our case because surgery was performed under local anesthesia and biochemistry, blood cell count, and coagulation tests were found to be normal.

Chronic hypotony with secondary macular edema can appear after any glaucoma surgical procedure [6], but in the context of our patient, this was also ruled out as the IOP was $12 \mathrm{~mm}$ $\mathrm{Hg}$ in the first week after surgery. In some cases, ODR can mimic a central retinal vein occlusion, but there was no retinal vessel tortuosity or optic nerve edema in fundoscopy, nor were there any delayed venous fillings on fluorescein angiography $[7,8]$.

\section{Conclusion}

ODR with macular edema after DS is an infrequent postoperative complication. It is more likely to occur in eyes with risk factors such as high myopia, even in the absence of a high preoperative IOP. This complication may result in significant morbidity, so both pre- and postoperative macular OCT are recommended during any filtering procedure to rule out macular edema that compromises visual acuity.

\section{Acknowledgments}

We acknowledge the contribution of all the staff of the Ophthalmology Department of Hospital Germans Trias i Pujol for their involvement in making the connection between clinical assistance and scientific work easier. 


\section{Statement of Ethics}

Our research was conducted ethically in accordance with the World Medical Association Declaration of Helsinki. Subjects gave their written informed consent required by current legislation for the publication of any personal data or images, and the study protocol was approved by the institute's committee on human research.

\section{Disclosure Statement}

The authors declare no conflicts of interest.

\section{Funding Sources}

The authors declare no funding sources.

\section{Author Contributions}

Jordi Loscos Arenas and Marc Carbonell Puig: visited the patient and worked on the manuscript. Laura Broc Iturralde: retina specialist who followed the patient. Pablo Diaz Aljaro: worked on and revised the manuscript, took the pictures, proofreading. Pau Romera Romero: revised the manuscript, correspondence author.

\section{References}

1 Mukkamala SK, Patel A, Dorairaj S, Mc Glynn R, Sidoti PA, Weinreb RN, et al. Ocular decompression retinopathy: a review. Surv Ophthalmol. 2013 Nov-Dec;58(6):505-12.

2 Kozobolis VP, Kalogianni E, Katsanos A, Dardabounis D, Koukoula S, Labiris G. Ocular decompression retinopathy after deep sclerectomy with mitomycin $\mathrm{C}$ in an eye with exfoliation glaucoma. Eur J Ophthalmol. 2011 May-Jun;21(3):324-7.

3 Al Obeidan SA, Osman EA, Mousa A, Al-Muammar AM, Abu El-Asrar AM. Long-term evaluation of efficacy and safety of deep sclerectomy in uveitic glaucoma. Ocul Immunol Inflamm. 2015 Feb;23(1):82-9.

4 Munteanu M, Munteanu G, Zolog I, Giuri S, Coviltir V, Stanca H, et al. [Ocular decompression retinopathy after combined deep sclerectomy and trabeculotomy]. Klin Monbl Augenheilkd. 2012 Aug;229(8):830-1. German.

5 Bui CM, Recchia FM, Recchia CC, Krammer JA. Optical coherence tomography findings in ocular decompression retinopathy. Ophthalmic Surg Lasers Imaging. 2006 Jul-Aug;37(4):333-5.

6 Kokame GT, de Leon MD, Tanji T. Serous retinal detachment and cystoid macular edema in hypotony maculopathy. Am J Ophthalmol. 2001 Mar;131(3):384-6.

7 Lee SJ, Lee JJ, Kim SD. Multiple retinal hemorrhage following anterior chamber paracentesis in uveitic glaucoma. Korean J Ophthalmol. 2006 Jun;20(2):128-30.

8 Obana A, Gohto Y, Ueda N, Miki T, Cho A, Suzuki Y. Retinal and subhyaloid hemorrhage as a complication of laser iridectomy for primary angle-closure glaucoma. Arch Ophthalmol. 2000 Oct;118(10):1449-51. 


\section{Case Reports in Ophthalmology}
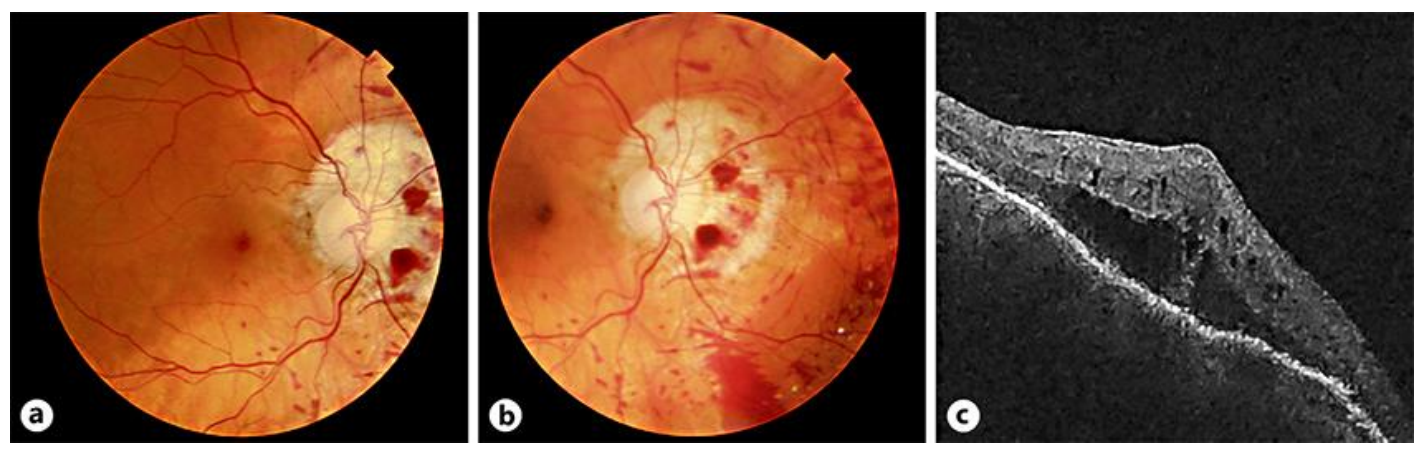

Fig. 1. a, b Fundus photography 3 weeks after deep sclerectomy. Posterior pole, peripapillary, and nasal retinal hemorrhages. c Corresponding optical coherence tomography showing severe macular edema.
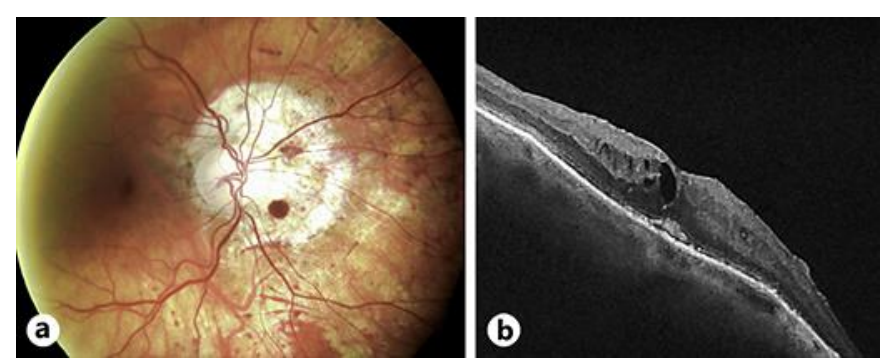

Fig. 2. a Fundus photography 2 months after deep sclerectomy. Peripapillary and posterior pole hemorrhages. b Corresponding optical coherence tomography showing intraretinal macular cysts.
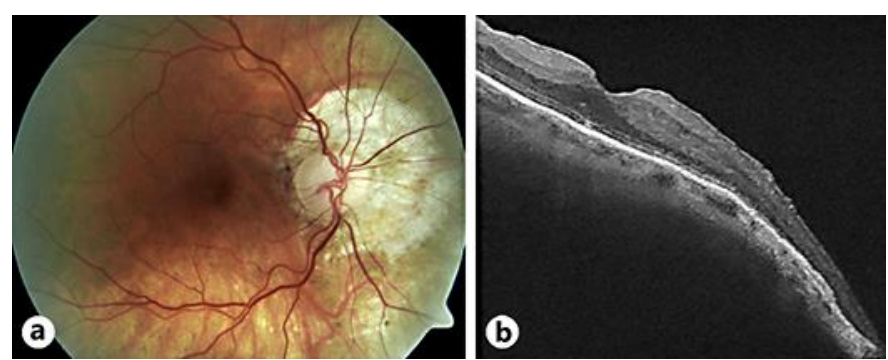

Fig. 3. a Fundus photography 6 months after deep sclerectomy. No retinal hemorrhages are seen. b Corresponding optical coherence tomography reveals resolved macular edema. 